\title{
The influence of the opening on the capacity of the community based on matlab software
}

\author{
Daoming Feng \\ College of Mathematics and Computer Science,Xinyu University ,Xinyu 338000, China
}

\begin{abstract}
This paper mainly studies the influence of community opening on road traffic. Combined with the reality of life, from the lane of the influencing factors, to find out several important factors, and accordingly the establishment of mathematical models for the community to put forward rationalization proposals.Firstly, the influence of different factors on the same index is selected by using the found data and analogy method. Through the analytic hierarchy process, the weight vector of each index is calculated by using MATLAB, and an appropriate evaluation index system is established.This paper establishes a mathematical model based on the equal relationship between traffic volume, traffic capacity and saturation before and after the opening of the district, and studies the influence of the opening of the district on the surrounding road traffic according to the basic traffic capacity and the crossing capacity of the access road.
\end{abstract}

\section{PROBLEM DESCRIPTION}

In recent years, with the national economic development level gradually increased, people have the ability to buy their own cars and rooms. Now most people are willing to go to the district to buy a house, first of all that the environment is better and close to the developed city, and that the surrounding area surrounded by the security of the enclosure is better from outside, but now because of the construction of the district more and more The greater the area occupied by the city, so that the road to building traffic less, causing frequent road congestion, so the public on the issue of whether the opening of the district can ease the traffic pressure to start a discussion, if the district can be opened by the The road inside and out of the district, but although the increase in the number of roads, but also led to other problems, such as will cause confusion within the district, the increase in traffic led to traffic congestion and traffic accidents increased probability.

In addition to security, the opening of the district is really open to optimize the road network structure, the following open to the surrounding roads on the impact of the following four questions:

Question 1: To evaluate the impact of open area on the surrounding road access to select the appropriate evaluation index system.

Question 2: to establish a mathematical model on the passage of vehicles, to study the opening of the district on the impact of the surrounding roads.

\section{Problem analysis}

For the first question: First, understand the changes before and after the opening of the district, the use of comparative law, the more likely to affect the traffic capacity of the indicators, select the impact of relatively large, due to the impact of factors are more common, for example, we can ignore external factors (Such as weather) and other unnecessary factors, the use of control variables to extract variables, residential open to the surrounding factors on the impact of the surrounding area through the study of our district road structure, community space size, traffic flow, residential parking, residential road structure between the data changes, The above factors are set up as the criterion layer and the target layer is the appropriate evaluation index system for the community. Therefore, we establish the model of the analytic hierarchy process, and then compare each other to determine the weight of the target layer. Finally, we use MATLAB to calculate the weight vector of each index, It will be drawn which indicators will affect the opening of the district and the impact of the size of the extent of the impact.

For the second question: First, the number of road around the road several cases of traffic analysis, the number of lanes is usually divided into two-way street, three-way street, four-way street, and analysis of residential road structure, residential road is a one-way street, and then we According to each road saturation is similar to the calculation of the district before and after the opening of the road saturation changes. There are Tshaped, Y-shaped, and round-shaped intersections at the intersection of the entrance and exit of the main road on the main road. However, we can only discuss the Tshaped, and then the three roads cross the road together, analyze the three cases Of the traffic density (hourly traffic) due to the main road, the traffic on the road will be different, assuming that the choice of road vehicles will not form a blockage, the initial determination of its saturation unchanged, the vehicle can run normally, and finally Compare the saturation of these three roads to determine the size of the impact on the surrounding roads, which is best.

\section{General assumptions and definitions}

\subsection{Assumptions}


- Assume that there is no vehicle failure or accident on the road.

- Suppose the road is flat, regardless of the slope effect.

- Assume that road vehicles are cars.

- Assume that the district is connected to the surrounding roads without traffic lights.

- Assuming that the speed of the surrounding road vehicle is constant, it is $30 \mathrm{~km} / \mathrm{h}$.

\subsection{Terms and definitions}

Table 1. Terms and Definitions

\begin{tabular}{|l|l|}
\hline $\mathrm{B}$ & Cell size \\
\hline $\mathrm{C}$ & District surrounding road structure \\
\hline $\mathrm{Q}$ & Traffic flow \\
\hline $\mathrm{D}$ & District parking volume \\
\hline $\mathrm{S}$ & District road structure \\
\hline $\mathrm{W}$ & Weight vector \\
\hline $\mathrm{h}$ & Indicates the number of lanes \\
\hline $\mathrm{N}$ & Road capacity \\
\hline $\mathrm{M}$ & Road saturation \\
\hline $\mathrm{K}$ & Road reduction factor \\
\hline $\mathrm{W}_{Z}$ & The weight vector of the cell space \\
\hline $\mathrm{W}_{\mathrm{C}}$ & $\begin{array}{l}\text { The Weight Vector of Residential Road } \\
\text { Structure }\end{array}$ \\
\hline $\mathrm{W}_{\mathrm{S}}$ & Traffic volume vector \\
\hline $\mathrm{W}_{\mathrm{D}}$ & The right vector of parking volume \\
\hline $\mathrm{W}_{3}$ & Weight vector of district road structure \\
\hline$\lambda_{\mathrm{max}}$ & The largest feature root \\
\hline $\mathrm{E}$ & Unit matrix \\
\hline $\mathrm{C}_{\mathrm{i}}, \mathrm{C}_{\mathrm{j}}$ & $\begin{array}{l}\text { The } \mathrm{i}, \mathrm{j} \text { elements of the criterion layer }(\mathrm{i}, \mathrm{j} \\
=1,2,3,4,5)\end{array}$ \\
\hline
\end{tabular}

\section{Establishment and solution of the model}

\subsection{The establishment of the hierarchy}

The decision-making problem is divided into two levels. According to the influence of the opening of the district on the surrounding roads, the corresponding evaluation indexes are set up. According to the information, the spatial size of the district $B$, the surrounding road structure (economic development area) Flow Q, parking volume $\mathrm{D}$, road structure $\mathrm{S}$ The main five factors affect the passage of the surrounding roads. The above factors are used as the second level of the level, and the pairwise comparison matrix of $5 \times 5$ is established according to the degree of the magnitude relationship of the influence of the second level on the first level. Finally, the pairwise comparison matrix is normalized The maximum eigenvector of each factor is calculated. If the matrix is consistent, then the maximum weight vector is used as the weight vector, and the evaluation index system with the first three effects is selected according to the order of the ratio. The fuzzy hierarchy is established according to the above method Analysis model one, as shown below:

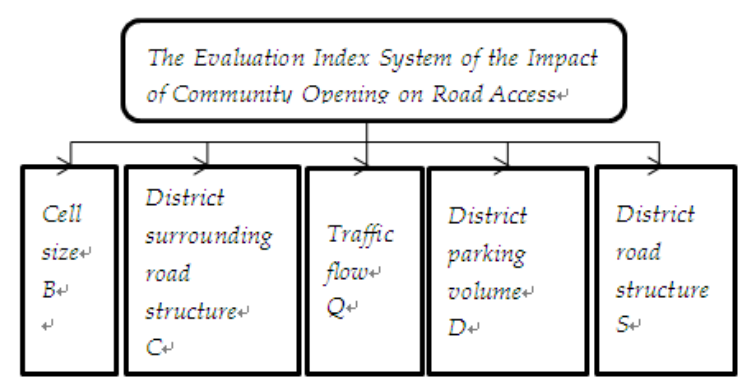

Fig. 1. Hierarchical model

\subsubsection{Determination of pairs of matrices}

With the development of the economy, people's living standards continue to improve, the number of residential quarters also will increase, the traffic volume also has an impact, in the decision-making factors, we can refer to the corresponding size of the data, from five factors were removed two $A$ factor out of comparison. Use $a_{i j}$ to represent the ratio of the $\mathrm{i}$-th and the $\mathrm{j}$-th factor, regardless of the influence of the other three factors, and so on.

As shown in Figure 1: Compare the impact of the second layer of five factors (Cell space size $\mathrm{C} 1$ 、 Community surrounding road structure $\mathrm{C} 2$ 、Traffic flow C3 、 Residential parking volume C4 、 Road structure C5 ) on the opening of the first layer of the district. From the second layer to select $A_{5}^{2}$ times two different factors to compare, when $C_{i}=C_{j}$ means that the selected for the same two factors for comparison. According to the proportion of reflexivity 1 , that is $a_{i i}=1, i=1,2,3,4,5$ are always established,used to indicate the impact on the factors of the previous level, The proportional result can be used as a comparison matrix

$$
A=\left(a_{i j}\right)_{5 \times 5}, a_{i j}>0, a_{j i}=1 / a_{i j}
$$

According to the comparison of the factors affecting the surrounding roads, we can form a pair of matrices, and let $a_{i j}=\frac{w_{i}}{w_{j}}$, then

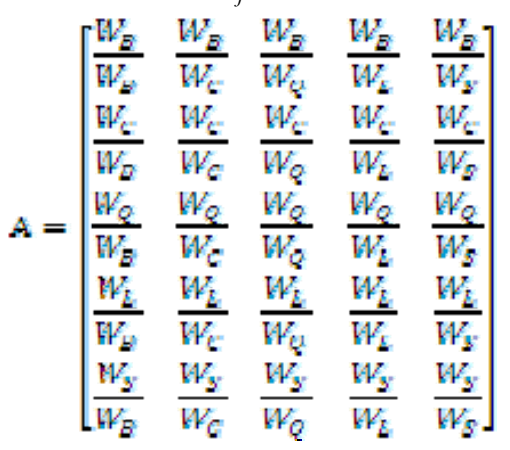


The pairwise comparison matrix is obtained by substituting the law and the proportional relationship:

$$
A=\left[\begin{array}{lllll}
1 & \frac{1}{3} & \frac{1}{5} & \frac{4}{7} & \frac{5}{8} \\
3 & 1 & \frac{4}{3} & \frac{5}{4} & \frac{7}{3} \\
5 & \frac{3}{4} & 1 & \frac{9}{5} & \frac{7}{5} \\
\frac{7}{4} & \frac{4}{5} & \frac{5}{9} & 1 & \frac{3}{5} \\
\frac{8}{5} & \frac{3}{7} & \frac{7}{5} & \frac{5}{3} & 1
\end{array}\right]
$$

\subsubsection{Compare scale determined}

(1) compare the two different nature of the indicators and come to a conclusion, we need to set a comparison scale to determine whether these indicators are realistic, according to the information and multi-party discussion, the indicators used to compare the use of two levels Analysis, determination of indicators:

Table 2. The meaning of each factor scale of Judgment matrix

\begin{tabular}{|c|c|}
\hline Scale aij & Meaning \\
\hline Similar to 1 & $C_{i}$ has the same effect as $C_{j}$ \\
\hline Similar to 3 & $C_{i}$ and $C_{j}$ slightly stronger \\
\hline Approximately & ${ }^{\mathrm{y}_{T}}$ The influence of $C_{i}$ and $C_{j}$ is strong \\
\hline Similar to 7 & $\begin{array}{l}\text { The effect of } C_{i} \text { and } C_{j} \text { is obviously } \\
\text { 'enhanced }\end{array}$ \\
\hline Similar to 9 & $\begin{array}{l}\text { The influence of } C_{i} \text { and } C_{j} \text { is } \\
\text { absolutely strong }\end{array}$ \\
\hline $\begin{array}{l}\text { Similar to } \\
2,4,6,8\end{array}$ & $\begin{array}{l}\text { The ratio of } C_{i} \text { to } C_{j} \text { is between the } \\
\text { two adjacent levels }\end{array}$ \\
\hline $\begin{array}{l}\text { Similar to the } \\
\text { reciprocal of } \\
\text { the above } \\
\text { numbers }\end{array}$ & $C_{i} / C_{j}$ becomes $C_{j} / C_{i}$ \\
\hline
\end{tabular}

(2) The proportion of the factors in the criterion layer is displayed according to the data of the search and can be fully accorded with the 1-9 scale. Therefore, it is only necessary to approximate the corresponding scale, for example, in matrix $\mathrm{A}$ The index is $7 / 4$ to about $1 / 2$, to determine its $1 / 2$ of the indicators, which can be derived from the other.

\subsubsection{Solve the weight vector and the maximum eigenvalue step}

According to the goal of the solution process, the use of step by step approach, starting from the bottom, according to the degree of correlation between the indicators listed in order, the specific steps are as follows: Calculate the weight vector $\mathrm{W}$ of each element on the target layer of the criterion layer.
According to the influence of the second level on the first level goal, the proportion of the two pairs is compared to the comparison matrix.

Find the eigenvalues $W_{i}(\mathrm{i}=1,2,3,4,5)$ under the eigenvalues are substituted by the formula

$$
(\quad|\lambda E-A|=0
$$

The eigenvectors of pairs of comparison matrices are calculated by MATLAB software.

The normalization of each eigenvector and the determination of the weight vector are as follows: the largest eigenvector (normalized) is chosen as the weight vector, and the normalized vector sum is 1 for accuracy. On the basis of this, we use the knowledge of MATLAB to calculate the maximum eigenvector as the weight vector $\mathrm{W}=\left[\begin{array}{lllll}0.1911 & 0.651 & 0.4777 & 0.3384 & 0.44367\end{array}\right]^{\mathrm{T}}$ as the weight vector of each factor.

Calculate the maximum eigenvalue $\lambda_{\max }$ of each element of the criterion layer for the target layer.

The eigenvalue is calculated from (4), and the largest eigenvalue of the eigenvector is selected from the calculated eigenvalue. The algorithm of the maximum eigenvalue can be realized by MATLAB, and $\lambda_{\max }=$ 5.0979 .

\section{Consistency test passed}

According to the search data, the maximum eigenvector $\lambda \geq n$ of the $n$-th pair of the comparison matrix $\mathrm{A}$ is shown, and $\mathrm{A}$ is a consistent matrix when $\lambda=n$. But the calculation is inevitable error, in order to better measure the consistency of this measure to establish a measure to determine the scope of consistency, when the characteristic root $\lambda$ than the order of the n-order matrix is much larger, to determine the degree of A serious degree of inconsistencies, so With the normalized eigenvector as the weight vector, the algorithm will be as follows

$$
C I=\frac{\lambda_{\max }}{n-1}
$$

As an indicator of consistency,when the CI is similar to 0 , it is determined that $\mathrm{A}$ is a consistency matrix; The greater the degree of inconsistency of CI, the more serious.In order to study the scope of the inconsistency,we need to find the maximum amount of measure to measure the inconsistency of A,Substituting the matrix into the above formula,using the MATLAB to calculate the maximum feature vector $\lambda_{\max }=5.0979$ into the above consistency index formula,substituting in (5) to get $\mathrm{CI}=0.02448$. To verify the consistency of the test,Here we introduce the randomness of the indicators

\begin{tabular}{|c|c|c|c|c|c|c|c|c|c|c|c|}
\hline $\mathrm{n}$ & 1 & 2 & 3 & 4 & 5 & 6 & 7 & 8 & 9 & 10 & 11 \\
\hline $\mathrm{R}$ & 0 & 0 & $\begin{array}{l}0.5 \\
8\end{array}$ & $\begin{array}{l}0.9 \\
0\end{array}$ & & $\begin{array}{l}1.2 \\
4\end{array}$ & $\begin{array}{l}1.3 \\
2\end{array}$ & $\begin{array}{l}1.4 \\
1\end{array}$ & $\begin{array}{l}1.4 \\
5\end{array}$ & $\begin{array}{l}1.4 \\
9\end{array}$ & $\begin{array}{l}1.5 \\
1\end{array}$ \\
\hline
\end{tabular}
RI,specific data as shown in Table III:

Table 3.Random consistency indicator RI values

The ratio of the random consistency index RI is called the consistency ratio $\mathrm{CR}$, that is, the consistency ratio CR 


$$
\mathrm{CR}=\mathrm{CI} / \mathrm{RI}<0.1
$$

It can be seen that the maximum eigenvector is within the allowable range, and the eigenvector can be used as the eigenvector after the eigenvector is the normalized vector of the eigenvector $\lambda=5.0979$

$$
\mathrm{W}=\left[\begin{array}{lllll}
0.1911 & 0.651 & 0.4777 & 0.3384 & 0.44367
\end{array}\right]^{\mathrm{T}}
$$

And then by (6), find the

$$
\mathrm{CI}=(5.0979-5) / 4=0.2448
$$

Since the established pairing matrix is 5 by 5 for the matrix in Table II, RI $=1.12$, use the formula (6) compute put

$$
\mathrm{CR}=\mathrm{CI} / \mathrm{RI}=0.02448 / 1.12=0.02186<0.1
$$

Conform to the set range, and consistency verification is passed as a weight vector.

A comprehensive evaluation of the indicators with the right vector,the basic steps are shown in Figure 2:

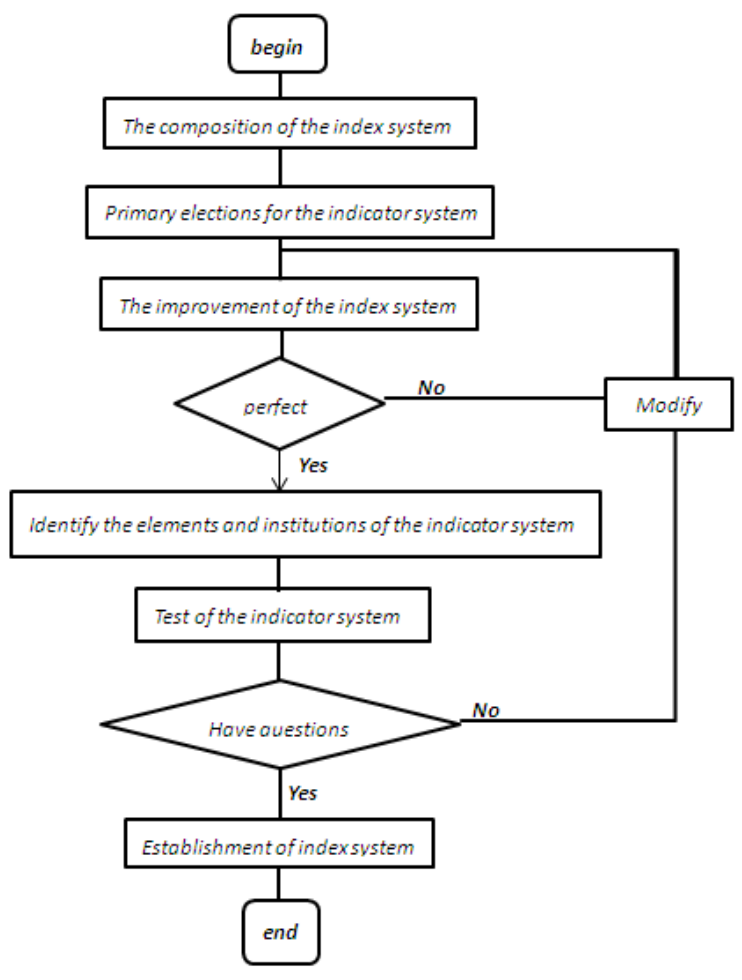

Fig. 2. Evaluation flow chart

Community open to the impact of road access we selected five evaluation index system, The weight vector of the district road structure is

$$
\mathrm{W}=\left[\begin{array}{lllll}
0.1911 & 0.651 & 0.4777 & 0.3384 & 0.44367
\end{array}\right]^{\mathrm{T}}
$$

In order to correspond to the above-mentioned index system, $\mathrm{WC}=0.6511, \mathrm{WQ}=0.4777, \mathrm{WS}=0.4436, \mathrm{WD}$ $=0.3384, \mathrm{WB}=0.1911$, which can be arranged from large to small.

It is concluded that the influence of the opening of the district on the traffic volume is arranged from large to small area for the surrounding road structure, traffic volume, community road structure, community parking volume and cell space size.

\subsection{Analyze the structure of the surrounding roads of the district to determine the road type of the vehicle}

As the structure of the plot of a variety of styles, where we take the road around the hospital for example, as shown in Figure 3: the district has a number of roads around, and each road structure is not the same, the road points For the one-way street, two-lane, three lanes, four lanes and other forms, and in the road junctions have to study the plane crossroads in the form of cross, T shaped, Y-shaped, round cross, multi-channel cross, In order to facilitate the study of the choice of T-word intersection, so we mainly consider the road type and the surrounding road structure and traffic flow of the three main factors, its open area on the surrounding road traffic will cause what impact and the intersection capacity The impact of the situation.

Based on the previous settings and information, we assume that the surrounding roads are composed of two different types. And assuming that there are hl lane in $\mathrm{n} 1$ roads around the area, and each road traffic is Q1; there are h2 lane in $n 2$ roads, and each road traffic flow is Q2. You can create a model:

$$
\left\{\begin{array}{l}
\frac{Q_{1}}{N_{1}\left(k_{1}+\cdots+k_{h 1}\right)}=\frac{Q_{2}}{N_{1}\left(k_{1}+\cdots+k_{h 2}\right)} \\
n_{1} Q_{1}+n_{2} Q_{2}=Q
\end{array}\right.
$$

In order to make the calculation more convenient, we can make:

$$
\left(k_{1}+\cdots+k_{h 1}\right)=r_{1},\left(k_{1}+\cdots+k_{h 2}\right)=r_{2}
$$

The equation can be reduced to :

$$
\left\{\begin{array}{l}
\frac{Q_{1}}{N_{1} r_{1}}=\frac{Q_{2}}{N_{1} r_{2}} \\
n_{1} Q_{1}+n_{2} Q_{2}=Q
\end{array}\right.
$$

From the (8) Can get

$$
\left\{\begin{array}{l}
Q_{1}=\frac{Q r_{1}}{n_{1} r_{1}+n_{2} r_{2}} \\
Q_{2}=\frac{Q r_{2}}{n_{1} r_{1}+n_{2} r_{2}}
\end{array}\right.
$$

According to the obtained Q1, Q2, the saturation can be obtained

$$
M_{1}=\frac{Q}{N_{1}\left(n_{1} r_{1}+n_{2} r_{2}\right)}
$$

$\mathrm{N} 1$ is the maximum traffic volume for a lane,M1 for road saturation, $k_{1}, \cdots, k_{h}$ is the ratio reduction coefficient of the road.

Because the road in the district is mainly two-way one-way road, but the number of single-street road number is different, so we can set the district has n3 twoway one-way street, if the open cell, the district road traffic for each road Q3, Each road flow becomes Q4, and the traffic volume of each car in the $\mathrm{h} 2$ lane becomes Q5. You can build the model from this:

$$
\left\{\begin{array}{l}
\frac{Q_{3}}{N_{1}}=\frac{Q_{4}}{N_{1} r_{1}}=\frac{Q_{5}}{N_{1} r_{2}} \\
n_{3} Q_{3}+n_{1} Q_{4}+n_{2} Q_{5}=Q
\end{array}\right.
$$

By the above equation together to obtain 


$$
\begin{aligned}
M_{2} & =\frac{Q}{N_{1}\left(n_{1} r_{1}+n_{2} r_{2}+n_{3}\right)} \\
M_{3} & =\frac{Q_{3}+Q_{4}}{N_{2}} \\
M_{4} & =\frac{Q_{3}+Q_{5}}{N_{3}}
\end{aligned}
$$

According to this model, the impact of the road on the surrounding roads can be studied by comparing the road saturation before and after the opening of the district and the change of the traffic volume of the main road and the intersection.

\section{Result analysis}

We get three conclusions:

(1) When the cell structure and the surrounding road structure does not change, the greater the traffic flow, the community before and after the saturation change the greater the change. But the greater the traffic flow at the same time, the community will make the intersection of the higher degree of saturation.

(2) when the cell structure and traffic flow does not change, the less the surrounding roads, residential area before and after the saturation changes the greater the change. But the less the surrounding roads, residential open will make the intersection of the higher saturation.

(3) When the surrounding road structure and traffic flow does not change, the more the internal road, the district before and after the saturation change the greater the change. And found the impact of the internal roads on the intersection.

Therefore, we have made the following recommendations based on these three points: we can give priority to open the surrounding roads within the road more of the district, because the number of internal roads on the intersection of the intersection will reduce the number of problems in the three tables have many types of district open Although the road saturation, But also will increase the burden of each intersection, when $M>1$, the traffic will be in a blocked state.

For the traffic flow of the district, the surrounding roads less of the district should be carefully considered whether open, although they can be a good way to alleviate the surrounding road traffic, but also likely to lead to crossing the block, so that all the surrounding roads blocked.

At the same time, we can rationalize the surrounding roads to improve the road capacity. Mitigate road traffic conditions.

\section{References}

1. Xiao zheng, zhu jiamin, xia Ming, building kang. Quantitative analysis on the influence of the opening of the community on the traffic capacity of surrounding roads[J].Journal of jiamusi university (natural science edition), 2015, 35(02):336-339.

2. Zhang heng, sun zhonggui, wu huimin. Mathematical modeling of the impact of the opening of the community on road traffic capacity [J]. Electronic technology and software engineering, 2017,(04):189. (2017-03-07).

3. Xiao jiqing. Evaluation study on the impact of the opening of the community on the impact of road capacity[J].Technology horizon, 2016,(26):198$+207$.

4. Li xiaoqing, zhu cunbin, li dongmei, Chen kai. Research on the influence of the opening of the community on the traffic capacity of surrounding roads[J].Journal of northern college of hebei (natural science edition), 2013, 33(09):39-43.

5. Liu Yang, zhang xueya, Yang zongyue, gong tenant. The impact model of open community on road traffic capacity[J].Technology innovation guide, 2015, 14(01):93-95.

6. Wang chai ping. Research on traffic network optimization in wuchang urban area based on Matlab simulation[D].Hubei university of technology, 2016.

7. Liu jiang. Selection of enterprise transportation based on analytic hierarchy process[D].University of international business and economics, 2006. 